\title{
Galerkin Approach for the Study of Urea Concentration Distribution in Artificial Kidney in Hemodialysis
}

\author{
Mina Gumanju', Dil Bahadur Gurung ${ }^{2}$ \\ ${ }^{1}$ Khwopa Higher Secondary School, Bhaktapur, Nepal \\ ${ }^{2}$ Department of Natural Science (Mathematics), School of Science, Kathmandu University, Nepal \\ Corresponding author: gumanju_mina@yahoo.com
}

Received: Nov 18, 2018

Revised: Dec 22, 2018

Accepted: Dec 23, 2018

\begin{abstract}
Hemodialysis is the process of purifying the blood which removes the waste product such as urea from blood through the semi-permeable membrane of dialyzer. In dialyzer, the rate of blood flow velocity is maintained around five times less than the dialyzed flow velocity during hemodialysis. The study focuses on the urea concentration distribution in the blood during dialysis using partial differential equation of diffusion process developed by J. N. Kapur, assuming blood is Newtonian, and laminar flow. The solution of urea concentration is obtained using Galerkin approximation method associated with appropriate boundary conditions.
\end{abstract}

Keywords: Diffusion process, Galerkin method, Urea concentration, Hemodialysis, Conjugate BVP

\section{Introduction}

Dialysis is primarily used to provide an artificial replacement for lost of kidney function due to renal failure. Dialysis treatment replaces waste removal like urea through the semipermeable membrane of artificial kidney which is performed by the dialyzer through diffusion process. Various dialyzers are in use; especially parallel plate dialyzer and hollow dialyzer. The first successful hemodialysis treatment in human was reported in 1944 [10]. A 67-year old woman in urea coma who regained consciousness after 11 hours of hemodialysis is considered to be first successful treated patient with Kolff's dialyzer in 1945 [3]. Before a mass transfer in dialyzers assumed that (a) the urea concentration of the solute in the dialyzed is zero or constant everywhere and that (b) the mass transfer resistance in the dialyzed is constant and may be added linearly to the resistance of membrane to give a composite resistance [2]. Very few articles are available working in mathematical model for hemodialysis. Cooney et al. [2] were first to work in this direction. They studied mass transfer in semi-infinite parallel plate dialyzers solving convective diffusion equation for blood and dialyzates. Their work was further extended by J. N. Kaptur in conjugate boundary value problem [5].

The present study is based on the model that consists of the partial differential equation defining the process of diffusion in artificial kidney, especially in circular duct dialyzer. The solution of the model equation is obtained in the form of urea concentration in blood and 
dialyzate by using the Galerkin approximation method by converting a continuous problem (such as a differential equation) to a discrete problem. In principle, it is equivalent of applying the method of variation of parameter to a function space, by converting the equation to a weak formulation. The Galerkin method of residuals is the most common method of calculating the global stiffness matrix in finite element method [1,9]. The aims of Galerkin's method is the production of a linear system of equations, and so we build its matrix form. The matrix of Galerkin equation is symmetric.

To apply Galerkin's method, we determine a sequence of functions satisfying the following conditions is determined.

- Each of the functions is continuously twice differentiable.

- Each function satisfies boundary conditions.

- The functions are linearly independent.

- The sequence of function is complete.

\section{Mathematical Equations}

The dialyzate is maintained more than blood flow in hemodialysis process. The flow of blood and dialyzate in a circular duct is considered to be axi-symmetric, i.e. it is symmetric about an axis. We use the cylindrical co-ordinates $(r, \theta, z)$, where the components of velocity, namely $v_{r}$, along the radial vector perpendicular to the axis, $v_{\theta}$ perpendicular to the axis and the radius vector, and $v_{z}$ parallel to the axis of $z$. For axi- symmetric case, we take $v_{\theta}=0$. We also consider the velocity of solute is in the fully developed flow and so $v_{z}=0$ (Fig. 1).

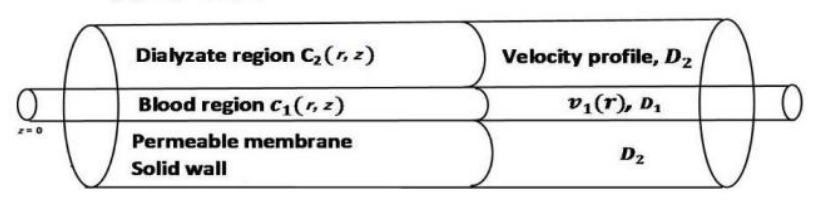

Fig 1: Circular dialyzer

The basic governing equation of urea concentration in an incompressible fully developed flow of fluid in a circular duct in cylindrical co-ordinate is given by [5]

$$
\frac{\partial C}{\partial t}+v(r, t) \frac{\partial C}{\partial z}=D\left(\frac{\partial^{2} C}{\partial r^{2}}+\frac{1}{r} \frac{\partial C}{\partial r}+\frac{\partial^{2} C}{\partial z^{2}}\right)
$$

where $C(r, z, t)$ is the concentration of urea in the fluid and $v(r, t)$ is the fluid velocity in the fully developed flow and $\mathrm{D}$ is the diffusivity of the fluid. The magnitude of the convective term as compared to the diffusion term is given by the dimensional Peclet number $P e=\frac{v_{m} R}{D}$, where $v_{m}$ is the maximum velocity in the fluid which occurs at the center of the blood vessel and $R$ is the radius of the duct. The value of $P e$ may be as large as 15,000 for the hemodialyzer [4]. So the longitudinal diffusion term can be neglected and then the above convection diffusion equation simplifies to steady case: 
Blood:

$$
D_{1}\left(\frac{\partial^{2} C_{1}}{\partial r^{2}}+\frac{1}{r} \frac{\partial C_{1}}{\partial r}\right)=v_{1}(r) \frac{\partial C_{1}}{\partial z}
$$

Dialyzate:

$$
D_{2}\left(\frac{\partial^{2} C_{2}}{\partial r^{2}}+\frac{1}{r} \frac{\partial C_{2}}{\partial r}\right)=v_{2}(r) \frac{\partial C_{2}}{\partial z}
$$

where $C_{1}(r, z)$ and $C_{2}(r, z)$ be the urea concentration in blood and dialyzate regions respectively, $D_{1}, D_{2}$ be their respective diffusivities in the blood and dialyzate regions, and $v_{1}(r), v_{2}(r)$ be the velocity distributions in blood and dialyzate regions determined from Newtonian fluid motion equation which depend on their viscosity coefficient, pressure gradient and the radii $r_{1}, r_{2}$ of inner and outer tubes.

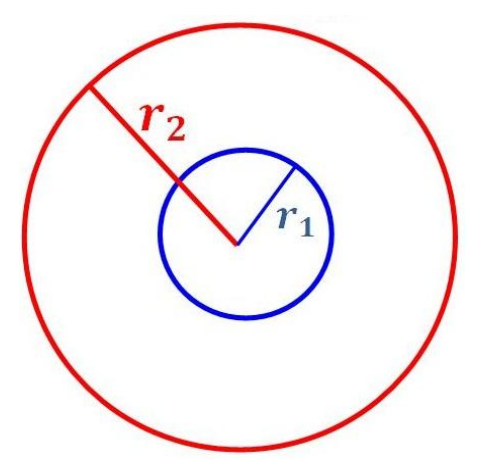

Fig. 2: Cross section of circular dialyzer

The approximation boundary conditions are:

$$
\begin{array}{ll}
\text { i) } & \frac{\partial C_{1}}{\partial r}=0 \text { at } r=0 \\
\text { ii) } & \frac{\partial C_{2}}{\partial r}=0 \text { at } r=r_{2} \\
\text { iii) } & -D_{1}\left[\frac{\partial C_{1}}{\partial r}\right]_{r=r_{1}}=-D_{2}\left[\frac{\partial C_{2}}{\partial r}\right]_{r=r_{1}}=P\left[C_{1}\left(r_{1}, z\right)-C_{2}\left(r_{2}, z\right)\right]=P\left[C_{1}(z)-C_{2}(z)\right]
\end{array}
$$

where $P$ is the permeability of the membrane of dialyzer.

\section{Solution of the Model Equations}

Applying the Laplace transform on convective diffusion equations for blood and dialyzate.

$D_{1}\left(\frac{\partial^{2} \bar{C}_{1}}{\partial r^{2}}+\frac{1}{r} \frac{\partial \bar{C}_{1}}{\partial r}\right)=v_{1}(r)\left(s \bar{C}_{1}-C_{10}\right)$

$D_{2}\left(\frac{\partial^{2} \bar{C}_{2}}{\partial r^{2}}+\frac{1}{r} \frac{\partial \bar{C}_{2}}{\partial r}\right)=v_{2}(r)\left(s \bar{C}_{2}-C_{20}\right)$

where $\bar{C}_{1}(r, s)$ and $\bar{C}_{2}(r, s)$ are the Laplace transform of $C_{1}(r, z)$ and $C_{2}(r, z), C_{10}$ and $C_{20}$ are initial urea concentration in blood and dialyzate. Similarly applying the Laplace transform on the boundary conditions (3) - (5)

$$
\text { i) } \frac{\partial \bar{C}_{1}}{\partial r}=0 \text { at } r=0
$$


ii) $\frac{\partial \bar{C}_{2}}{\partial r}=0$ at $r=r_{2}$

iii) $\quad-D_{1}\left[\frac{\partial \bar{C}_{1}}{\partial r}\right]_{r=r_{1}}=-D_{2}\left[\frac{\partial \bar{C}_{2}}{\partial r}\right]_{r=r_{1}}=P\left[C_{1}(s)-C_{2}(s)\right]$

where $\bar{C}_{1}(s)=\bar{C}_{1}\left(r_{1}, s\right), \bar{C}_{2}(s)=\bar{C}_{2}\left(r_{1}, s\right)$ at $r=r_{1}$

To apply Galerkin approach, we try with the following solutions for equations (6) and (7)

$\bar{C}_{1}(r, s)-\bar{C}_{2}(s)=\sum_{i=1}^{n}\left(a_{i}+b_{i} r^{2 i}\right) f_{i}(s)=\sum_{i=1}^{n} h_{i}(r) f_{i}(s)$

$\bar{C}_{2}(r, s)-\bar{C}_{1}(s)=\sum_{i=1}^{n}\left(A_{i}+B_{i}\left(r-r_{1}\right)^{2 i}\right) F_{i}(s)=\sum_{i=1}^{n} H_{i}(r) F_{i}(s)$

where the sequence of functions $f_{i}(s)$ and $F_{i}(s), 1 \leq i \leq n$ satisfy the conditions to apply Galerkin approach. The above two tried solutions consists $(2 n+2)$ functions

$f_{1}(s), f_{2}(s), f_{3}(s), \ldots, f_{n}(s) ; \quad F_{1}(s), F_{2}(s), F_{3}(s), \ldots, F_{n}(s)$ and $\bar{C}_{1}(s), \bar{C}_{2}(s)$ and the $4 n$ constants.

$$
\begin{gathered}
a_{1}, a_{2}, a_{3}, \ldots, a_{n} ; b_{1}, b_{2}, b_{3}, \ldots b_{n} ; A_{1}, A_{2}, A_{3}, \ldots, A_{3} ; B_{1}, B_{2}, B_{3}, \ldots, B_{n} . \\
-D_{1} \frac{\partial}{\partial r} \sum_{i=1}^{n}\left[a_{i}+b_{i} r^{2 i}\right]_{r=r_{1}} f_{i}(s)=-D_{2} \frac{\partial}{\partial r} \sum_{i=1}^{n}\left[A_{i}+B_{i}\left(r-r_{1}\right)^{2 i}\right]_{r=r_{1}} F_{i}(s) \\
P\left[\bar{C}_{1}(s)-\bar{C}_{2}(s)\right]=-P\left[\bar{C}_{2}(s)-\bar{C}_{1}(s)\right] \\
\text { If we put, } \quad-2 i b i D_{1} r_{1}^{2 i-1}=C, \quad-2 i B i D_{2}\left(r-r_{1}\right)^{2 i-1}=C \\
P\left(a_{i}+b_{i} r^{2 i}\right)=C, \quad-P\left[A_{i}+B_{i}\left(r-r_{1}\right)^{2 i}\right]=C
\end{gathered}
$$

We find values of $a_{i}, b_{i}, A_{i}, B_{i}$, where $C$ is a constant value, and using (14) in (13).

$$
\sum_{i=1}^{n} f_{i}(s)=\sum_{i=1}^{n} F_{i}(s)=Q(s) \text {, say }
$$

For simplicity our calculation, we assume $Q(s)=\frac{K}{s}$ and then using equation (16) and (17)

$$
\bar{C}_{1}(s)-\bar{C}_{2}(s)=\frac{C}{P} Q(s), \quad \bar{C}_{2}(s)-\bar{C}_{1}(s)=-\frac{C}{P} Q(s)
$$

Both equations give, $L\left[C_{1}(z)-C_{2}(z)\right]=\frac{C}{P} Q(s)$

Then,

$$
Q(s)=\frac{P}{C}\left[\frac{C_{1}(z)-C_{2}(z)}{s}\right]
$$

Thus from equation (17), (18), (19), we get

$$
\sum_{i=1}^{n} f_{i}(s)=\sum_{i=1}^{n} F_{i}(s)=\left[\frac{C_{10}-C_{20}}{s}\right]
$$

Without loss of generality, if we assume $C=P$,

$a_{i}=1+\frac{r_{1} P}{2 i D_{1}}=1+\frac{S h_{w}}{2 i} ; \quad b_{i}=-\frac{r_{1} P}{2 i D_{1} r_{1}^{2 i}}=-\frac{1}{2 i} \frac{S h_{w}}{2 i}$

$A_{i}=-1-\frac{\left(r_{2}-r_{1}\right) P}{2 i D_{2}}=-1-\frac{s h_{W}^{\prime}}{2 i} ; \quad B_{i}=\frac{1}{2 i} \frac{\left(r_{2}-r_{1}\right) P}{D_{2}\left(r_{2}-r_{1}\right)^{2 i}}=-\frac{1}{2 i} \frac{S h_{W}^{\prime}}{\left(r_{2}-r_{1}\right)^{2 i}}$

Then, the equation (20) to (22) 
$a_{i}>0, b_{i}<0, A_{i}<0, B_{i}>0, h_{i}\left(r_{1}\right)>0, H_{i}\left(r_{1}\right)<0$

The orthogonality relation gives,

$\int_{0}^{r_{1}} R_{1}(r, s) h_{j}(r) r d r=0$, for all $j=1,2,3, \ldots, n$

$\int_{r_{1}}^{r_{2}} R_{2}(r, s) H_{j}\left(r-r_{2}\right) d r=0$, for all $j=1,2,3, \ldots, n$

Then, $\quad \sum_{i=1}^{n}\left(a_{i j}+s b_{i j} r^{2 i}\right) f_{i}(s)=p_{j}\left[s \bar{C}_{2}(s)-C_{10}\right]$

$$
\sum_{i=1}^{n}\left(A_{i j}+s B_{i j}\left(r-r_{1}\right)^{2 i}\right) F_{i}(s)=P_{j}\left[s \bar{C}_{1}(s)-C_{20}\right]
$$

where, $\quad a_{i j}=-D_{1} \int_{0}^{r_{1}} \frac{1}{r} \frac{\partial}{\partial r}\left(\frac{r \partial h_{i}}{\partial r}\right) h_{j}(r) d r$,

$$
\begin{aligned}
b_{i j} & =\int_{0}^{r_{1}} v_{1}(r) h_{i}(r) h_{j}(r) r d r, \quad p_{j}=\int_{0}^{r_{1}} v_{1}(r) h_{j}(r) r d r, \\
A_{i j} & =D_{2} \int_{r_{1}}^{r_{2}} \frac{1}{r} \frac{\partial}{\partial r}\left(\frac{r \partial H_{i}}{\partial r}\right) H_{j}\left(r-r_{2}\right) d r, \\
B_{i j} & =\int_{r_{1}}^{r_{2}} v_{2}(r) H_{i}(r) H_{j}\left(r-r_{2}\right) d r, \quad P_{j}=-\int_{0}^{r_{1}} v_{2}(r) h_{j}\left(r-r_{2}\right) d r
\end{aligned}
$$

Here $\left(a_{i j}\right)$ and $\left(b_{i j}\right)$ are symmetric matrices with all positive elements. If $v_{2}(r)>0$, then the dialyzate flow is in same direction as blood flow and if $v_{2}(r)<0$, then the dialyzate flow is in opposite direction of the blood flow. $B_{i j}$ is symmetric and has all negative elements and if $v_{2}(r)>0$ and has all positive elements if $v_{2}(r)<0$. All $p_{j}$ 's are negative and $P_{j}$ 's are negative if $v_{2}(r)>0$ and positive if $v_{2}(r)<0$. Let us denote

$$
\begin{aligned}
& g_{i}(s)=\frac{f_{i}(s)}{s \bar{C}_{2}(s)-C_{10}} \\
& G_{i}(s)=\frac{F_{i}(s)}{s \overline{C_{1}}(s)-C_{20}}
\end{aligned}
$$

After simplification, we get

$$
\begin{aligned}
& \sum_{i=1}^{n}\left(a_{i j}+s b_{i j}\right) g_{i}(s)=p_{j} ; j=1,2,3, \ldots, n \\
& \sum_{i=1}^{n}\left(A_{i j}+s B_{i j}\right) G_{i}(s)=P_{j} ; j=1,2,3, \ldots, n
\end{aligned}
$$

From these equations we can obtain $g_{i}(s)$ and $G_{i}(s)$, and we find that each is rational function of $s$ whose numerator is a polynomial of $(n-1)$ th degree and denominator is a polynomial of $\mathrm{n}^{\text {th }}$ degree, and is denoted by $R(n-1, n)$. From equations $(20),(27),(28)$, we obtain

$$
\sum g_{i}(s)=\frac{\sum f_{i}(s)}{s \bar{C}_{2}(s)-C_{10}} \text { and } s \bar{C}_{2}(s)-C_{10}=\frac{C_{10}-C_{20}}{s \sum g_{i}(s)}
$$

$\sum G_{i}(s)=\frac{\sum F_{i}(s)}{s \bar{C}_{1}(s)-C_{10}}$ and $s \bar{C}_{1}(s)-C_{10}=\frac{C_{10}-C_{20}}{s \sum g_{i}(s)}$

Both $\sum g_{i}(s), \sum G_{i}(s)$ are $R(n-1, n)$ functions and

$$
\bar{C}_{2}(s)=\frac{C_{10}}{s}+\frac{C_{10}-C_{20}}{s^{2} \sum g_{i}(s)}, \quad \bar{C}_{1}(s)=\frac{C_{20}}{s}+\frac{C_{10}-C_{20}}{s^{2} \sum G_{i}(s)}
$$

which are $R(n, n+1)$ functions. Using equation (31) and (32), we have 


$$
\begin{array}{lrl}
g_{i}(s)=\frac{f_{i}(s)}{s \bar{C}_{2}(s)-C_{10}}, & s \bar{C}_{2}(s)-C_{10}=\frac{f_{i}(s)}{g_{i}(s)} \\
G_{i}(s)=\frac{F_{i}(s)}{s \bar{C}_{1}(s)-C_{20}}, & s \bar{C}_{1}(s)-C_{20}=\frac{F_{i}(s)}{G_{i}(s)}
\end{array}
$$

Putting the value of $g_{i}$ from equation (27) in equation (31)

$\frac{C_{10}-C_{20}}{s \sum g_{i}(s)}=\frac{f_{i}(s)}{g_{i}(s)^{\prime}}, \quad f_{i}(s)=\frac{C_{10}-C_{20}}{s \sum g_{i}(s)} \frac{g_{i}(s)}{\sum g_{i}(s)}$

Similarly, putting the value of $G_{i}$ from equation (28) in equation (32)

$$
\frac{C_{10}-C_{20}}{s \sum G_{i}(s)}=\frac{F_{i}(s)}{G_{i}(s)}, \quad F_{i}(s)=\frac{C_{10}-C_{20}}{s} \frac{G_{i}(s)}{\sum G_{i}(s)}
$$

Both $f_{i}(s)$ and $F_{i}(s)$ are $R(n-1, n)$ functions, we then obtain $C_{1}(z), C_{2}(z), C_{1}(r, z), C_{2}(r, z)$ using the following Laplace Inverses ,

$$
\begin{aligned}
& C_{1}(z)=L^{-1}\left(\bar{C}_{1}(s)\right), \quad C_{2}(z)=L^{-1}\left(\bar{C}_{2}(s)\right) \\
& C_{1}(r, z)-C_{2}(z)=\sum_{i=1}^{n}\left(a_{i}+b_{i} r^{2 i}\right) L^{-1}\left(f_{i}(s)\right) \\
& C_{2}(r, z)-C_{1}(z)=\sum_{i=1}^{n}\left(A_{i}+B_{i}\left(r-r_{1}\right)^{2 i}\right) L^{-1}\left(F_{i}(s)\right)
\end{aligned}
$$

The above equations give for urea concentration in blood and dialyzate.

\section{Numerical Results and Discussion}

In our simulation results, the integrals for $A_{i j}$ and $B_{i j}$ are evaluated using Simpson's $\frac{1}{3}$ rule. A parallel plate dialyzers have two side by side $15 \mathrm{~cm} \times 90 \mathrm{~cm}$ blood path per layer, that is about $0.54 \mathrm{~m}^{2}$ of membrane per layer. According to Cooney et al. [2], blood channel height is normally about $0.03 \mathrm{~cm}$ under dynamic conditions. Blood flow rates in dialyzer is about $100 \mathrm{~cm}^{3} / \mathrm{min}$ per layer. Dialyzate flow rate is considered $500 \mathrm{~cm}^{3} / \mathrm{min}$ per layer. Cooney et al. has experimentally observed that urea concentration in blood side around the permeable membrane is approximately five times more than in dialyzate side. This results that urea concentration in blood side at any instant must be more than the urea in dialyzate side. The urea diffuses through semi-permeable membrane of dialyzer within the temperature range $27^{\circ} \mathrm{C}-37^{\circ} \mathrm{C}$. Within these temperature range, the investigators [10] suggested the membrane diffusivity values $0.265 \times 10^{-5} \mathrm{~cm}^{2} / \mathrm{sec}$ at $27^{\circ} \mathrm{C}$ and $37^{\circ} \mathrm{C}$ respectively. Cooney et al. [10] has considered the inner radius of dialyzer $0.03 \mathrm{~cm}$, and the total radius of the dialyzer as $0.06 \mathrm{~cm}$. They observed that $D_{1}=10^{-5} \mathrm{~cm}^{2} / \mathrm{sec}, D_{2}$ and is greater than $D_{1}$.

Cooney et al. [2] has experimentally observed that the urea concentration in blood side around the permeable membrane is approximately five times more than in dialyzate side. The observation is exhibited in the simulated results of Fig. 1 and 2. In our simulated result, we observe same behavior in urea concentration distribution at the same radial distance measured from $r=0$ in blood side and $r=r_{1}$ from dialyzate side. This is due to the fact of the adjustment of flow in blood and dialyzate side during hemodialysis process. In hemodialysis process, concentration is assumed to be maximum at the axis, i.e. $r=0$. It then decreases 
towards the semi- permeable membrane. This shows that the urea concentration in blood is left five times less than after it passing through the semi-permeable membrane. These results satisfying the condition that blood velocity is five times less than the velocity dialyzate.

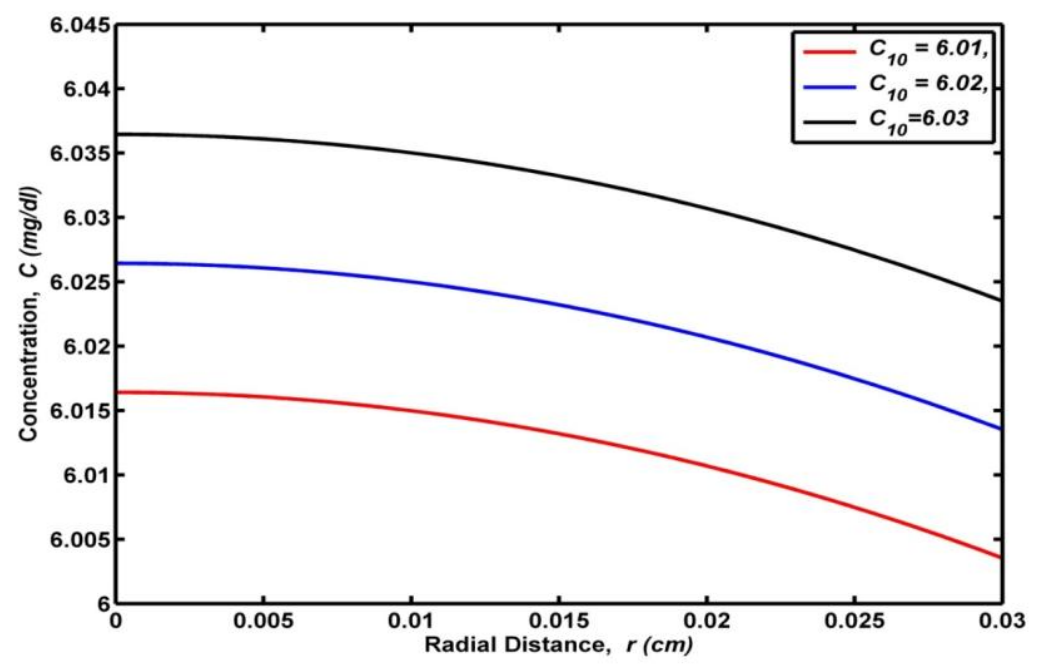

Fig. 3: Urea concentration in blood

For various values of urea concentration in blood and assuming the other parameter values as: $v_{1}=100 \mathrm{~cm}^{3} / \mathrm{min}, v_{2}=500 \mathrm{~cm}^{3} / \mathrm{min}, r_{1}=0.03 \mathrm{~cm}, r_{2}=0.06 \mathrm{~cm}, P=0.285 \times 10^{-5} \mathrm{~cm}^{2} / \mathrm{sec}$, $D_{1}=10^{-5} \mathrm{~cm}^{2} / \mathrm{sec}, D_{2}=1.5 \times 10^{-5} \mathrm{~cm}^{2} / \mathrm{sec}$

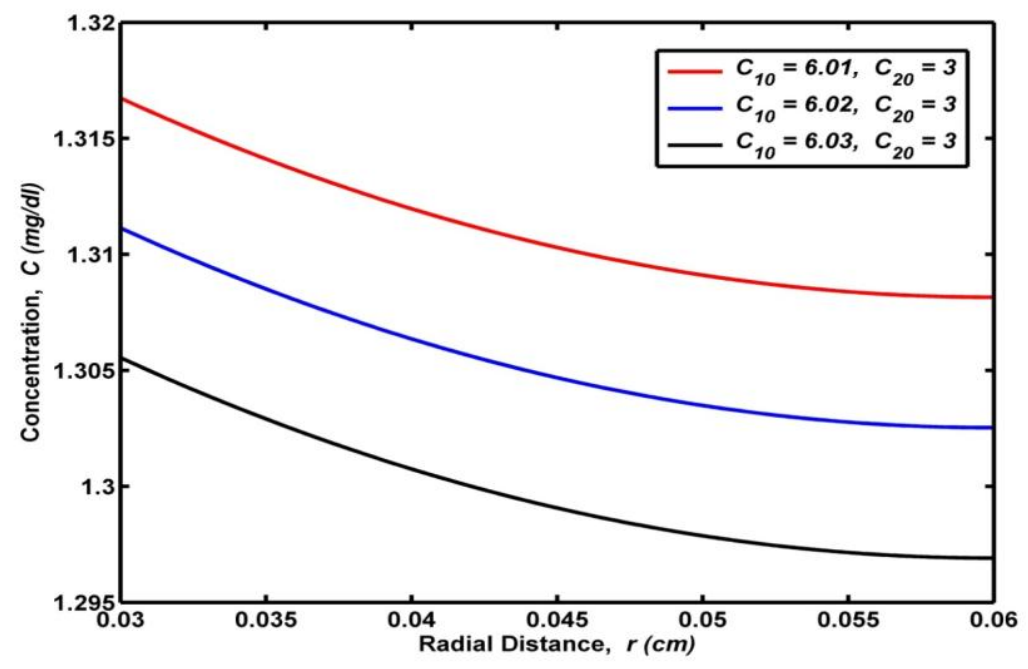

Fig. 4: Urea concentration in dialyzate

Assuming the parameter values: $v_{1}=100 \mathrm{~cm}^{3} / \min , v_{2}=500 \mathrm{~cm}^{3} / \mathrm{min}, r_{1}=0.03 \mathrm{~cm}$, $r_{2}=0.06 \mathrm{~cm}, P=0.285 \times 10^{-5} \mathrm{~cm}^{2} / \mathrm{sec}, D_{1}=10^{-5} \mathrm{~cm}^{2} / \mathrm{sec}, D_{2}=1.5 \times 10^{-5} \mathrm{~cm}^{2} / \mathrm{sec}$, 
$C_{10}=$ initial condition of urea concentration in blood, $C_{10}=$ initial condition of urea concentration in dialyzate.

\section{Conclusion}

From the study, it is observed the differences between the urea concentration in blood and dialyzate. It is due to decrease in the urea concentration in blood and increase in urea pass through the semi-permeable membrane in dialyzate during dialysis process. The blood velocity in dialyzate governs the velocity of urea concentration of dialyzate.

Acknowledgement: The first author acknowledges UGC Nepal for providing financial support under M.Phil Research Support 2073/2074 scheme to prepare this article.

\section{References}

[1] Ciarlet PG (1978), The finite element method for elliptic problems, Springer, North-Holland.

[2] Cooney O.David, E. James Davis, and Shin -Seung Kim (1974), Mass transfer in parallel plate dialyzer, a conjugate boundary value problem, The Chemical Engineering, Elsevier Sequoia S.A., Lausanne. Printed in the Netherlands 8, 213-222.

[3] Cooney O. David, E. James Davis, and Shin -Seung Kim (1974), Mass transfer in parallel plate dialyzer, a conjugate boundary value problem, The Chemical Engineering 8, 213-20.

[4] Kapur JN (1985), Mathematics model in biology and medicine, Affiliated East-West Press Private Limited, New Delhi.

[5] Kapur JN (1981), Solution of the conjugate boundary value problem of mass transfer in circular duct dialyzers by using Galerkin's method, Indian J. pure applied mathematics, 12(2): 163-174.

[6] Kolav SD.and Van der Linden WE(1992), Analysis of transient laminar mass transfer in parallel plate dialyzer, Analytic chimica Acta., 257: 331-342.

[7] Kolav SD and Van der Linden WE (1992), Analysis of transient laminar mass transfer in parallel plate dialyzer, Analytic chimica Acta., 257: 317-329.

[8] Kolff Willem-Kidney, http://www.kidney dialysis, Org.uk/inventor-of-dialysis.htm.

[9] Mikhlin SG (1964), Variational in mathematics, physics,Pergamon press.

[10] Shinaberger JH (2001), Quantiation of dialysis, Hostorical Prespective, Sem in Dialysis, 4(14): 238-245.

[11] Yeh HM and Hsu CW (2013), Analysis of mass transfer in parallel-flow dialyzer with internal recycle for improved performance, Chemical Engineering and Processing, 13: 1-28. 